\title{
Medical treatment of pulmonary hypertension in adults with congenital heart disease: updated and extended results from the International COMPERA-CHD Registry
}

\begin{abstract}
Ann-Sophie Kaemmerer ${ }^{1}$, Matthias Gorenflo ${ }^{2}$, Dörte Huscher ${ }^{3}$, David Pittrow ${ }^{4}$, Peter Ewert ${ }^{1}$, Christine Pausch, Marion Delcroix ${ }^{6}$, Hossein A. Ghofrani ${ }^{7}$, Marius M. Hoeper ${ }^{8}$, Rainer Kozlik-Feldmann ${ }^{9}$, Andris Skride ${ }^{10}$, Gerd Stähler $^{11}$, Carmine Dario Vizza ${ }^{12}$, Elena Jureviciene ${ }^{13}$, Dovile Jancauskaite ${ }^{13}$, Lina Gumbiene ${ }^{13}$, Ralf Ewert $^{14}$, Ingo Dähnert ${ }^{15}$, Matthias Held ${ }^{16}$, Michael Halank ${ }^{17}$, Dirk Skowasch ${ }^{18}$, Hans Klose ${ }^{19}$, Heinrike Wilkens ${ }^{20}$, Katrin Milger ${ }^{21}$, Christian Jux ${ }^{22}$, Martin Koestenberger ${ }^{23}$, Laura Scelsi ${ }^{24}$, Eva Brunnemer ${ }^{25}$, Michael Hofbeck ${ }^{26}$, Silvia Ulrich ${ }^{27}$, Anton Vonk Noordegraaf ${ }^{28}$, Tobias J. Lange ${ }^{29}$, Leonhard Bruch $^{30}$, Stavros Konstantinides ${ }^{31}$, Martin Claussen ${ }^{32}$, Judith Löffler-Ragg ${ }^{33}$, Hubert Wirtz ${ }^{34}$, Christian Apitz $^{35}$, Rhoia Neidenbach ${ }^{1}$, Sebastian Freilinger ${ }^{1}$, Attila Nemes ${ }^{36}$, Christian Opitz $^{37 \#}$, Ekkehard Grünig ${ }^{38 \#}$, Stephan Rosenkranz ${ }^{39 \#}$
\end{abstract}

${ }^{1}$ Deutsches Herzzentrum München, Klinik für angeborene Herzfehler und Kinderkardiologie, München, Technische Universität München, and Deutsches Zentrum für Herz-Kreislauf-Forschung (DZHK), Munich Heart Alliance, Munich, Germany; ${ }^{2}$ Universitätsklinikum Heidelberg, Zentrum für Kinder- und Jugendmedizin, Angelika-Lautenschläger-Klinik, Heidelberg, Germany; ${ }^{3}$ Institute of Biometry and Clinical Epidemiology, and Berlin Institute of Health, Charité-Universitätsmedizin, Berlin, Germany; ${ }^{4}$ Medical Faculty, Institute for Clinical Pharmacology, Technical University, Dresden, Germany; ${ }^{5}$ Epidemiological Centre, GWT-TUD GmbH, Dresden, Germany; ${ }^{6}$ UZ Leuven, Campus Gasthuisberg, Department of Pneumology, Leuven, Belgium; ${ }^{7}$ Universitätsklinikum Gießen und Marburg GmbH, Medizinische Klinik II/V, Gießen, Germany; ${ }^{8}$ Medizinische Hochschule Hannover, Abt. Pneumologie, Hannover, Germany; ${ }^{9}$ Universitäres Herzzentrum Hamburg, Klinik und Poliklinik für Kinderherzmedizin und Erwachsene mit angeborenen Herzfehlern, Hamburg, Germany; ${ }^{10}$ Riga Stradins University, Clinical University Hospital, Riga, Lativa; ${ }^{11}$ Klinik Löwenstein, Medizinische Klinik I, Löwenstein, Germany; ${ }^{12}$ Pulmonary Hypertension Center, Department of Clinical Anestesiologic and Cardiovascular Sciences, University of Rome La Sapienza, Rome, Italy; ${ }^{13}$ Faculty of Medicine of Vilnius University, Referal Centre of Pulmonary Hypertension, Vilnius University Hospital Santaros Klinikos, Vilnius, Lithuania; ${ }^{14}$ Universitätsmedizin Greifswald, Zentrum für Innere Medizin, Klinik und Poliklinik für Innere Medizin B, Greifswald, Germany; ${ }^{15}$ Herzzentrum Leipzig GmbH, Klinik für Kinderkardiologie, Leipzig, Germany; ${ }^{16}$ Med. Klinik mit Schwerpunkt Pneumologie und Beatmungsmedizin, Klinikum Würzburg Mitte, Standort Missioklinik, Würzburg, Germany; ${ }^{17}$ Universitätsklinikum Carl Gustav Carus der Technischen Universität Dresden, Medizinische Klinik und Poliklinik I, Dresden, Germany; ${ }^{18}$ Universitätsklinikum Bonn, Medizinische Klinik und Poliklinik II, Innere Medizin - Kardiologie/Pneumologie, Bonn; ${ }^{19}$ Abteilung für Pneumologie, Zentrum für Onkologie, Universitätsklinikum Hamburg-Eppendorf, Hamburg, Germany; ${ }^{20}$ Universitätsklinikum des Saarlandes, Innere Medizin V, Homburg, Germany; ${ }^{21}$ LMU Klinikum, Medizinische Klinik und Poliklinik V, München, Germany; ${ }^{22}$ Kinderherzzentrum und Zentrum für angeborene Herzfehler, Justus-Liebig-Universität, Zentrum für Kinderheilkunde, Abteilung Kinderkardiologie, Gießen, Germany; ${ }^{23}$ LKH - Univ. Klinikum Graz, Universitätsklinik für Kinder- und Jugendheilkunde, Abteilung für Pädiatrische Kardiologie, Graz, Austria; ${ }^{24}$ Fondazione IRCCS Policlinico San Matteo University of Pavia, Pavia, Italy; ${ }^{25}$ Medizinische Universitätsklinik (Krehl-Klinik), Klinik für Kardiologie, Angiologie und Pneumologie (Innere Medizin III), Heidelberg, Germany; ${ }^{26}$ Universitätsklinik für Kinder- und Jugendmedizin Tübingen, Kinderkardiologie, Pulmologie, Intensivmedizin, Tübingen, Germany; ${ }^{27}$ Universitätsspital Zürich, Klinik für Pneumologie, Zürich, Switzerland; ${ }^{28}$ Amsterdam UMC, Vrije Universiteit Amsterdam, dept of Pulmonary Medicine, Amsterdam, Netherlands; ${ }^{29}$ Universitätsklinikum Regensburg, Medizinische Klinik und Poliklinik II, Regensburg, Germany; ${ }^{30}$ Unfallkrankenhaus Berlin, Klinik für Innere Medizin, Berlin, Germany; ${ }^{31}$ Universitätsmedizin Mainz, Center for Thrombosis and Hemostasis, Mainz, Germany; ${ }^{32}$ LungenClinic Grosshansdorf, Fachabteilung Pneumologie, Großhansdorf, Germany; ${ }^{33}$ Universitätsklinik für Innere Medizin II, Innsbruck, Austria; ${ }^{34}$ Universitätsklinikum Leipzig, Medizinische Klinik und Poliklinik I, Abteilung für Pneumologie, Leipzig, Germany; ${ }^{35}$ Universitätsklinik für Kinder- und Jugendmedizin, Sektion Pädiatrische Kardiologie, Ulm, Germany; ${ }^{36}$ University of Szeged, Department of Medicine, Szeged, Hungary; ${ }^{37}$ DRK Kliniken Berlin Westend, Klinik für Innere Medizin, Schwerpunkt Kardiologie, Berlin, Germany; ${ }^{38}$ Thoraxklinik Heidelberg gGmbH, Zentrum für Pulmonale Hypertonie, Heidelberg, and German Centre for Lung Research, Heidelberg, Germany; ${ }^{39}$ Universitätsklinik Köln - Herzzentrum, Klinik III für Innere Medizin, Köln, Germany

Contributions: (I) Conception and design: AS Kaemmerer; (II) Administrative support: M Gorenflo, D Huscher, D Pittrow, MM Hoeper, C Opitz, 
E Grünig, S Rosenkranz; (III) Provision of study materials or patients: All authors; (IV) Collection and assembly of data: All authors; (V) Data analysis and interpretation: AS Kaemmerer, M Gorenflo, D Huscher, D Pittrow, MM Hoeper, C Opitz, E Grünig, S Rosenkranz; (VI) Manuscript writing: All authors; (VII) Final approval of manuscript: All authors.

\#These authors contributed equally to this work.

Correspondence to: Ann-Sophie Kaemmerer, MD. Department of Congenital Heart Disease and Pediatric Cardiology, Deutsches Herzzentrum München, Technische Universität München, München, Germany. Email: Kaemmerer@dhm.mhn.de; askaemmerer@gmail.com.

Background: Pulmonary arterial hypertension (PAH) is common in congenital heart disease (CHD). Because clinical-trial data on PAH associated with CHD (PAH-CHD) remain limited, registry data on the long-term course are essential. This analysis aimed to update information from the COMPERA-CHD registry on management strategies based on real-world data.

Methods: The prospective international pulmonary hypertension registry COMPERA has since 2007 enrolled more than 10,000 patients. COMPERA-CHD is a sub-registry for patients with PAH-CHD

Results: A total of 769 patients with PAH-CHD from 62 specialized centers in 12 countries were included into COMPERA-CHD from January 2007 through September 2020. At the last follow-up in 09/2020, patients [mean age $45.3 \pm 16.8$ years; 512 (66\%) female] had either post-tricuspid shunts $(\mathrm{n}=359 ; 46.7 \%)$, pre-tricuspid shunts $(\mathrm{n}=249 ; 32.4 \%)$, complex CHD ( $\mathrm{n}=132 ; 17.2 \%)$, congenital left heart or aortic valve or aortic disease $(n=9 ; 1.3 \%)$, or miscellaneous CHD ( $n=20 ; 2.6 \%)$. The mean 6 -minute walking distance was $369_{ \pm} 121 \mathrm{~m}$, and $28.2 \%, 56.0 \%$, and $3.8 \%$ were in WHO functional class I/II, III or IV, respectively $(12.0 \%$ unknown). Compared with the previously published COMPERA-CHD data, after 21 months of followup, the number of included PAH-CHD patients increased by 91 (13.4\%). Within this group the number of Eisenmenger patients rose by 39 (16.3\%), the number of "Non-Eisenmenger PAH" patients by 45 (26.9\%). Currently, among the 674 patients from the PAH-CHD group with at least one follow-up, $450(66.8 \%)$ received endothelin receptor antagonists (ERA), 416 (61.7\%) PDE-5 inhibitors, 85 (12.6\%) prostacyclin analogues, and $36(5.3 \%)$ the sGC stimulator riociguat. While at first inclusion in the COMPERA-CHD registry, treatment was predominantly monotherapy $(69.3 \%)$, this has shifted to favoring combination therapy in the current group (53\%). For the first time, the nature, frequency, and treatment of significant comorbidities requiring supportive care and medication are described.

Conclusions: Analyzing "real life data" from the international COMPERA-CHD registry, we present a comprehensive overview about current management modalities and treatment concepts in PAH-CHD. There was an trend towards more aggressive treatment strategies and combination therapies. In the future, particular attention must be directed to the "Non-Eisenmenger PAH" group and to patients with complex CHD, including Fontan patients.

Trial Registration: www.clinicaltrials.gov, study identifier: Clinicaltrials.gov NCT01347216

Keywords: Congenital heart disease (CHD); Eisenmenger syndrome; pulmonary hypertension; registry; targeted treatment

Submitted Jun 02, 2021. Accepted for publication Jul 30, 2021.

doi: $10.21037 / \mathrm{cdt}-21-351$

View this article at: https://dx.doi.org/10.21037/cdt-21-351

\section{Introduction}

All patients with congenital heart disease (CHD) are considered as chronic heart disease patients, regardless of whether they have native disease or have been interventionally/surgically treated $(1,2)$. Owing to medical advances in recent decades, in the industrialized world, up to $97 \%$ of patients with CHD now survive to adulthood (3). However, it is common for residua, sequelae, and cardiac or non-cardiac comorbidities to develop that adversely 
impact the course of the disease and increase morbidity and mortality (4). Pulmonary vascular disease (PVD) and pulmonary hypertension $(\mathrm{PH})$ are the most important longterm complications, besides defect-specific residua, heart failure, and cardiac arrhythmias. The most severe form of PVD in CHD is Eisenmenger syndrome, which develops as a complication in a primary left-to-right shunt lesion (5).

For a long time, severe forms of $\mathrm{PH}$ were considered largely unresponsive to medical therapy, and lung transplantation combined with intracardiac repair or heartlung transplantation were the final choice. However, the development of pulmonary vasoactive drugs has expanded the therapeutic armamentarium and favorably influenced the quality of life and survival of many affected patients (6). Despite the fact that several drugs are at present approved for the treatment of pulmonary arterial hypertension (PAH), there are only limited data on their use in CHD (7).

The aim of the current study was to evaluate and update data on targeted pharmacological management of patients with CHD and PVD from the COMPERA registry, which enrolls patients with all forms of $\mathrm{PH}$ (8).

\section{Methods}

This study was an extended and updated analysis of data from the international COMPERA registry (study identifier: Clinicaltrials.gov NCT01347216) on PH (8). At the time of the present analysis (September 01, 2020), 62 centers from 12 European countries (Austria, Belgium, Germany, Greece, Hungary, Italy, Latvia, Lithuania, the Netherlands, Slovakia, Switzerland, and the United Kingdom) participated in the sub-registry on CHD (COMPERACHD). Inclusion criteria for the present analysis were the diagnosis of $\mathrm{PH}$ in $\mathrm{CHD}$, a minimum age of 18 years, any targeted PAH medication, including endothelin receptor antagonists (ERA), phosphodiesterase-5 inhibitors (PDE5i), the soluble guanylate cyclase (sGC) stimulator riociguat, or prostacyclin analogues (including selexipag as an oral prostacyclin agonist), and written informed consent. Exclusion criteria were lack of cognitive competence to consent to participate in the research project or refusal to consent.

The PAH associated with CHD (PAH-CHD) group comprised patients on targeted PAH medication for PVD with Eisenmenger's syndrome, non-Eisenmenger patients with severe PAH based on a CHD ("Non-EM-PAH"), and patients who had undergone a modified Fontan operation. The "Non-EM-PAH" group included adults with CHD after previous reparative surgery or interventions for an intra- or extracardiac systemic-to-pulmonary shunt, as well as CHD associated with prevalent systemic-to-pulmonary shunts, or PAH with small/coincidental defects. In addition, patients with Eisenmenger syndrome without targeted PAH medication were also included.

\section{Data collection and documentation}

Patients were enrolled consecutively, and internetbased documentation included cardiac diagnoses, patient demographics, type of PAH according to Dana Point/Nice classification, surgical or interventional treatments, clinical status (including functional class, 6-min walk test, selected laboratory variables, and quality of life), cardiac and noncardiac comorbidities, and detailed information on PAH medication and supportive medication. Data at entry were supplemented by data from routine readmissions or in the case of a predetermined clinical event (worsening functional class, PAH-related hospitalization, unanticipated changes in PAH therapy, transplantation, death, or other serious adverse events). Plausibility and completeness checks, queries, and/or on-site monitoring were undertaken to secure high data quality.

The registry has been approved by the institutional review boards of all contributing centers and written informed consent was obtained from all participating patients before the start of documentation (lead ethics committee at Technical University Dresden, Germany: approval EK 129052007 as of 22 May 2007). The study was conducted in accordance with the Declaration of Helsinki (as revised in 2013). Guidelines on good pharmacoepidemiological practice (GPP) and data protection guidelines were followed.

\section{Statistical analysis}

The study population was descriptively presented as percentage, median with interquartile range (IQR), or mean \pm standard deviation. Categorical variables were compared using $\chi^{2}$-test or Fisher's exact test. Group differences, after testing for normal distribution, were assessed by $t$-test. Otherwise, the 2-sided Mann-Whitney test was used. A P value of $<0.05$ was considered significant. Adjustment for multiple testing was omitted. Data analysis was performed using SPSS 24.0 (IBM Inc., Armonk, NY, USA).

The author(s) of this manuscript have certified that they comply with the Principles of Ethical Publishing in the 
Table 1 Comparison of epidemiologic data from the COMPERA-CHD-Registry as of December 1, 2018 and September 2020 (21-month interval)

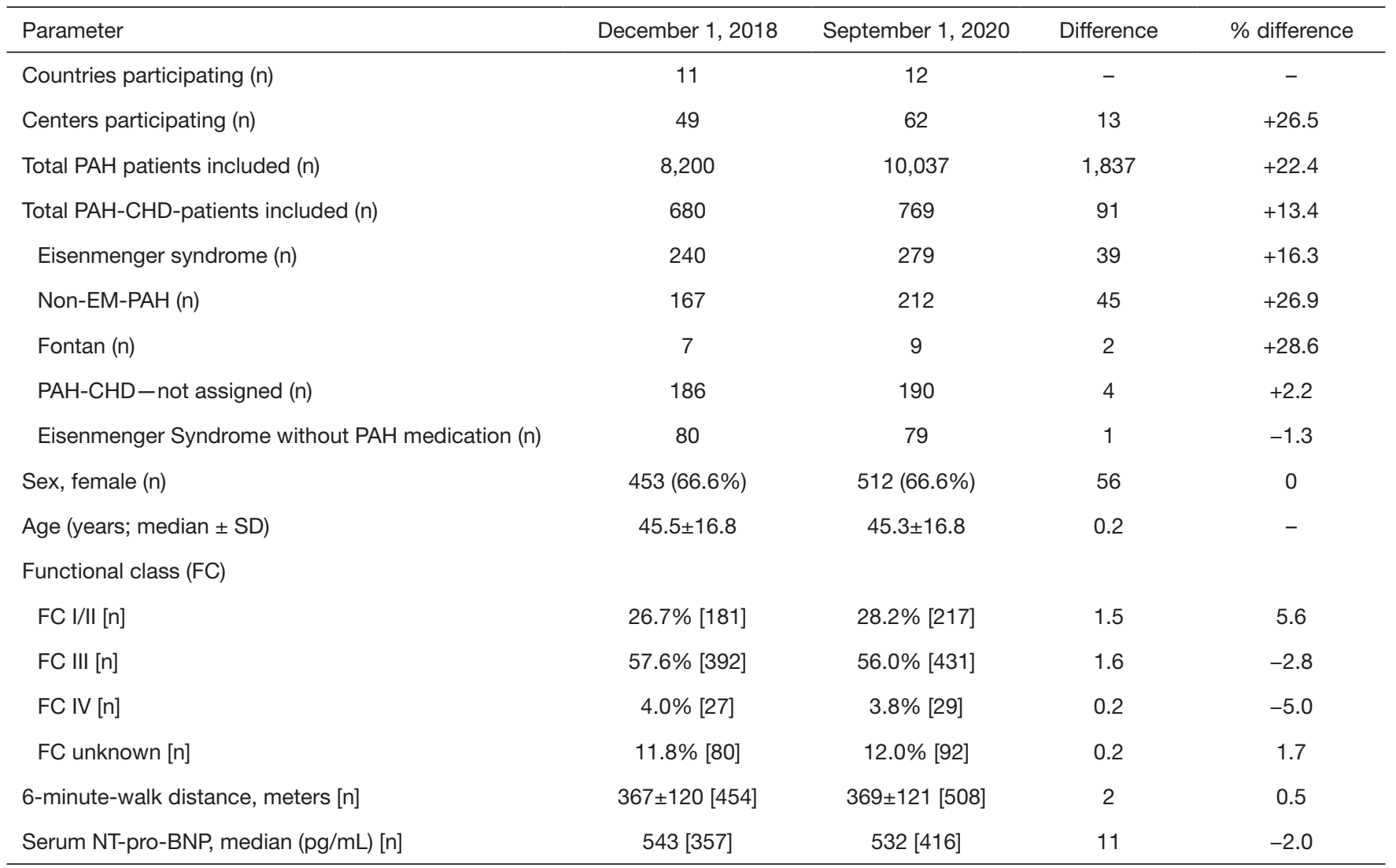

EM, Eisenmenger syndrome; FC, functional class according to Perloff; Non-EM-PAH, patients with congenital heart disease-associated pulmonary arterial hypertension but without Eisenmenger syndrome; $\mathrm{PAH}$, pulmonary arterial hypertension; $\mathrm{PAH}-\mathrm{CHD}$, congenital heart disease-associated pulmonary arterial hypertension; n, number.

American Fournal of Cardiology (9).

\section{Results}

As of September 1, 2020, a total of 10,265 patients were enrolled in the COMPERA registry at 62 centers; 10,037 cases were adults and 228 were children (Table 1). Within the entire group, 769 patients had CHD (7.7\%).

A subgrouping was possible in 579 of these patients: 279 were on targeted PAH therapy because of Eisenmenger syndrome, 212 because of CHD and severe PVD which, however, did not meet the criteria for Eisenmenger syndrome ("Non-Eisenmenger-PAH"), and 9 because of PVD after Fontan surgery. In addition, 79 patients with Eisenmenger syndrome but without targeted PAH therapy were included (Table 1).

Thus, compared with the initial description of the
COMPERA-CHD collective (as of December 1, 2018) (8), over a 21-month period in the PAH-CHD-group, the number of Eisenmenger patients rose by 39 (16.3\%), and the number of "Non-Eisenmenger-PAH" patients increased by $45(26.9 \%)$. The number of Fontan patients and untreated Eisenmenger patients changed only marginally (Table 1). At the same time, the number of included $\mathrm{PAH}$ patients in COMPERA increased by 1,837 (22.4\%).

\section{Type of CHD}

According to the frequency of occurrence, CHD could be assigned to five main groups: post-tricuspid shunts; pretricuspid shunts; complex anomalies; congenital anomalies of the left heart, aortic valve, and aorta; and CHD that could not be further categorized (Table 2).

At last follow-up, patients had either post-tricuspid 
Table 2 Assignment of patients to different subgroups according to type of congenital heart defect as of December 1, 2018 and September 2020 (21-month interval)

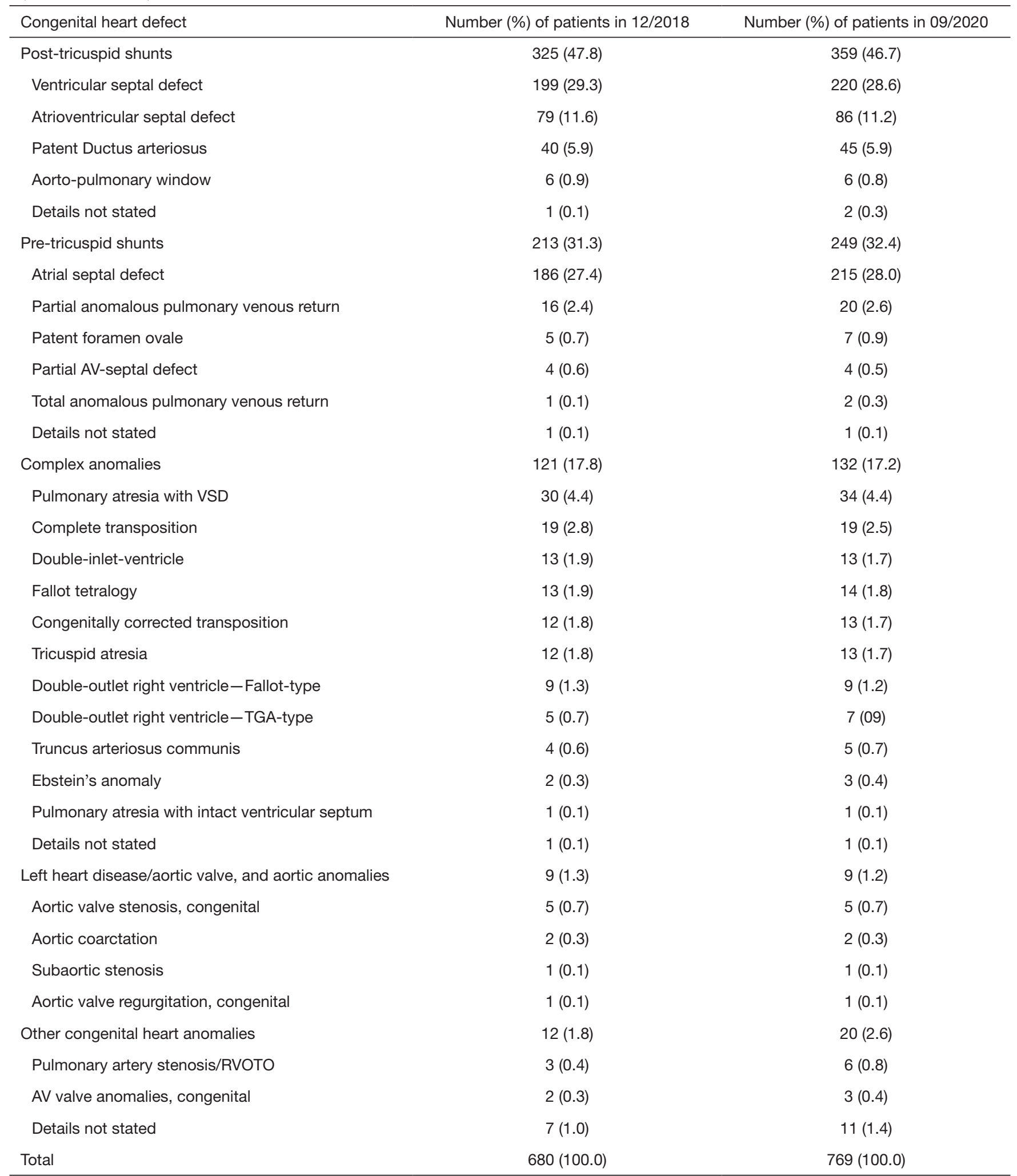

$\mathrm{AV}$, aortic valve; VSD, ventricular septal defect; TGA, transposition of the great arteries; RVOTO, right ventricular outflow tract disruption. 
shunts $(\mathrm{n}=359 ; 46.7 \%)$, pre-tricuspid shunts $(\mathrm{n}=249$; $32.4 \%)$, complex CHD ( $\mathrm{n}=132 ; 17.2 \%)$, congenital anomaly of the left heart, aortic valve, or aorta $(n=9 ; 1.3 \%)$, or miscellaneous other types of CHD ( $\mathrm{n}=20 ; 2.6 \%)$ (Table 2). Overall, the most prevalent CHDs were ventricular septal defects, atrial septal defects, atrioventricular septal defects, patent ductus arteriosus, and pulmonary atresia with ventricular septal defect. Compared with the 2018 COMPERA data, there were no significant differences in the type of CHD included (8).

\section{Patient demographics and baseline characteristics}

In the current analysis, 512 patients $(66.6 \%)$ were female and 257 patients (33.4\%) were male. A similar sex ratio was found in the subgroup analyses (Table 1). The mean age of all PAH-CHD patients was $45.3 \pm 16.8$ years. The 9 patients with Fontan circulation were considerably younger $(36.0 \pm 11.6$ years $)$ (Table 1). More than half of the patients were in their third, fourth, or fifth decade of life $(n=429,55.8 \%)$. Of the remaining patients, $169(22.0 \%)$ were younger than 30 years and $171(22.2 \%)$ were in the 6th decade of life. In comparison to the previous COMPERA analysis (8), there were no significant differences regarding gender or age distribution. Key demographic and clinical baseline data in comparison with the 2018 data are provided in Tables 1-3.

\section{Additive clinical data}

A total of 431 patients $(56.0 \%)$ were in functional class III; the exercise capacity, measured as 6-minute walking distance $(6 \mathrm{MWD})$, averaged $369 \pm 121$ meters in the actual analysis (Table 1). In comparison with the COMPERA data from 2018, there was no significant difference.

Furthermore, analysis of the 2020 data revealed the importance of cardiac and non-cardiac comorbidities found in 289 of 656 patients (44.1\%); in particular, coronary artery disease, arterial hypertension, thromboembolism, sleep apnea syndrome, diabetes mellitus, or thyreopathies. Atrial fibrillation or flutter was described in 86 patients $(11.2 \%)$, and syncope during the course of the disease was reported in 42 of 388 patients $(10.8 \%)$. A pacemaker was needed by 25 of 418 patients (6.0\%). Trisomy-21 was present in 100 of 448 patients (Figure 1).

\section{PAH therapy}

All currently available drugs for targeted PAH therapy were used in the patients with CHD included in COMPERA. Only in individual cases were calcium channel blockers part of the medication.

Among the 674 PAH-CHD patients with at least one follow-up, ERA were used in 450 (66.8\%), a PDE5i in 416 $(61.7 \%)$, prostanoids in $85(12.6 \%)$, and a sGC stimulator in $36(5.3 \%)$. In most diagnostic groups, ERA were used more frequently than PDE5i, prostanoids, or riociguat. Only in the very small Fontan cohort ( $\mathrm{n}=8$ with follow-up) was a PDE5i the preferred drug class (prescribed in seven out of eight patients; Figure 2). Within drug classes, bosentan $(\mathrm{n}=210)$ was more frequently used than ambrisentan $(\mathrm{n}=73)$, or macitentan $(n=163)$ as the ERA, while sildenafil $(n=280)$ was used more frequently than tadalafil $(n=136)$ as the PDE5i $(\mathrm{n}=416)$.

At the time of inclusion in the COMPERA-CHD registry, treatment was predominantly monotherapy $(69.3 \%)$ in the overall population. Monotherapy was also preferred in the subgroups with Eisenmenger syndrome (65\%), those with non-Eisenmenger syndrome with PAH (73.7\%), and in Fontan patients (75\%). The largest percentage of patients on combination therapy was seen in the Eisenmenger group $(35.0 \%)$.

In the 674 patients who had at least one follow-up visit after enrollment in COMPERA-CHD, therapy shifted in favor of combination therapy (53\%). The percentage of patients on combination therapy increased to $62.1 \%$ in the subgroup with Eisenmenger syndrome, to $46.9 \%$ in the group with "Non-Eisenmenger-PAH"; and also in the small Fontan group.

Even compared with the 2018 treatment data, there was another three percent increase in patients taking combination therapy.

\section{Additive and supportive medical treatment}

In addition to targeted PAH medication, additive or supportive medical treatment was deemed necessary in the majority of patients. Specifically, the following drugs were administered: diuretics ( $\mathrm{n}=279$ of $586 ; 47.6 \%$ ), beta blockers $(\mathrm{n}=164$ of $578 ; 28.4 \%)$, ACE inhibitors or angiotensin receptor blockers (ARBs) ( $\mathrm{n}=100$ of $570 ; 17.5 \%)$, digitalis $(\mathrm{n}=84$ of $573 ; 14.7 \%)$, or class III antiarrhythmics $(\mathrm{n}=32$ of $568 ; 5.6 \%)$.

The use of anticoagulants varied considerably with regard to the type of agent used in the different disease groups. Antiplatelet agents, vitamin $\mathrm{K}$ antagonists, nonvitamin $\mathrm{K}$ antagonists (NOAC), and heparins were used. 


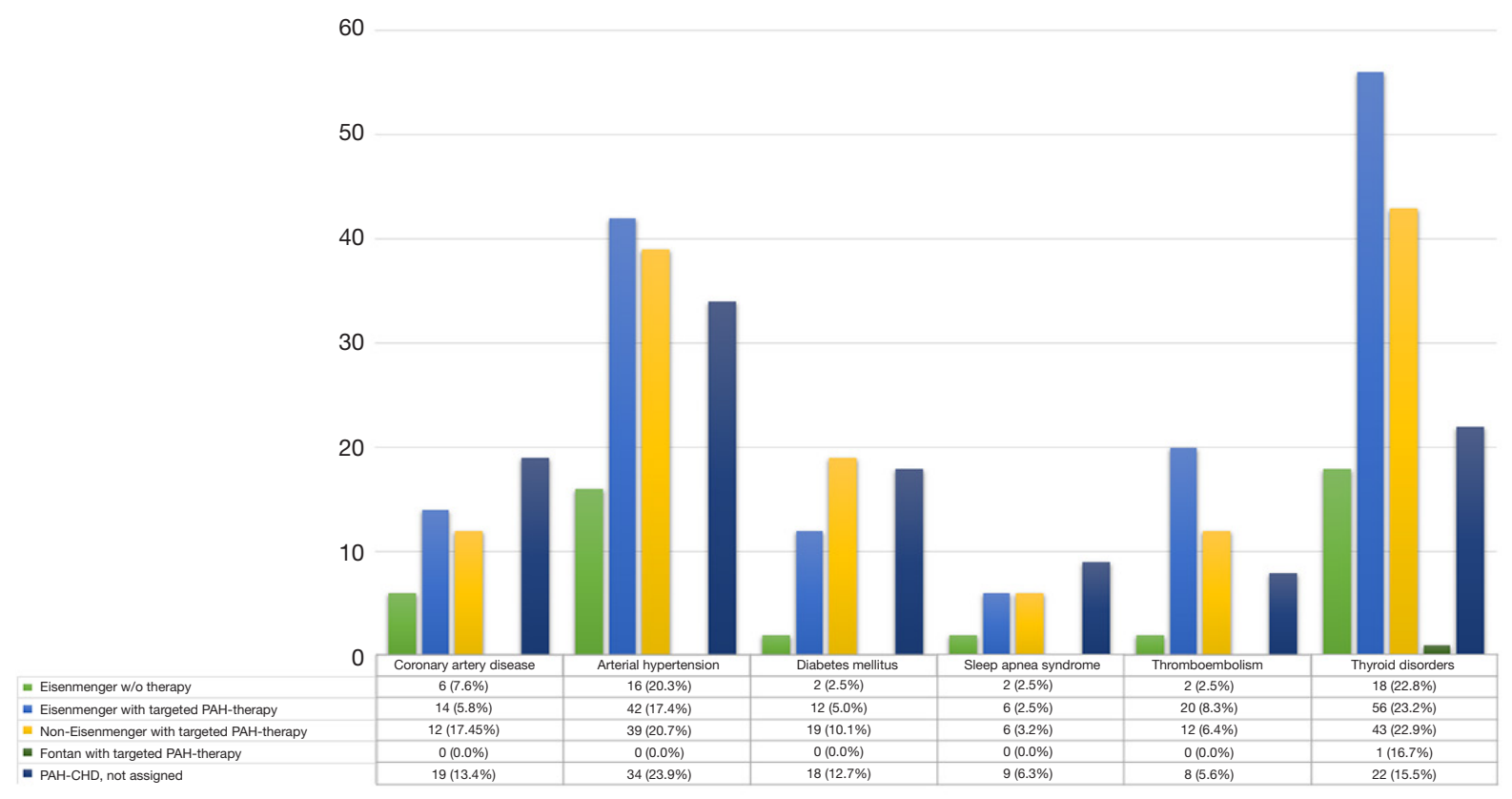

Figure 1 Clinically relevant comorbidities within the different groups of PAH associated with CHD. CHD, congenital heart disease; EM, Eisenmenger syndrome; Non-EM-PAH, patients with congenital heart disease-associated pulmonary arterial hypertension but without Eisenmenger syndrome; PAH, pulmonary arterial hypertension.

Of the 674 patients who had at least one follow-up visit, at the time of enrollment in the COMPERA registry, a large proportion of patients were without anticoagulation: 49 in the therapy-naive Eisenmenger group (68.1\%), 133 in the treated Eisenmenger group (54.7\%), and 88 in the "Non-Eisenmenger-PAH" group (49.2\%). In contrast, five out of eight patients with Fontan circulation were on oral anticoagulation with vitamin $\mathrm{K}$ antagonists. During the follow-up period, only minor changes in the percentage distribution of anticoagulant medication were observed (Figure 3).

\section{Discussion}

PAH develops in $5-10 \%$ of patients with CHD, and accounts for $34-42 \%$ of all PAH cases (10-12). Despite improved therapeutic measures and a decreasing incidence in industrialized countries, PAH-CHD will remain a problem, especially in lower-income countries and in the future, the number of patients with complex CHD and pulmonary vascular disease, including those with Fontan circulation, will significantly increase (13).

In contrast to other forms of $\mathrm{PAH}$, only limited data exist on targeted, diseaseoriented therapy for PAH-CHD
$(6,7,14)$.

Because most data on PAH therapy in CHD are derived from small, uncontrolled studies that typically include only a few patients and short observation periods, it is important to utilize data from registries that include patients with PAH-CHD; and to update these data regularly (15-19).

Nearly all other PH registries in Europe and the United States that also include CHD comprise all types of group 1 patients (PAH) but differentiate less precisely between clinical subtypes, treatment characteristics, and outcomes of distinct patient groups (20-30).

As one of the world's largest $\mathrm{PH}$ registries, the international COMPERA registry provides comprehensive, clinically relevant information on targeted PAH treatment, including patients with PAH-CHD. Meanwhile, the COMPERA-CHD subregistry represents the largest registry of PAH in CHD ( $\mathrm{n}=769)$, allowing sophisticated epidemiological and clinical analyses because of its sample size and detailed documentation (8).

The increase in the numbers of PAH-CHD patients is relatively less than the number of patients with other forms of PAH, whose numbers have increased by $22 \%$ over the same period. Within the past two years, the number of patients with PAH-CHD in COMPERA 


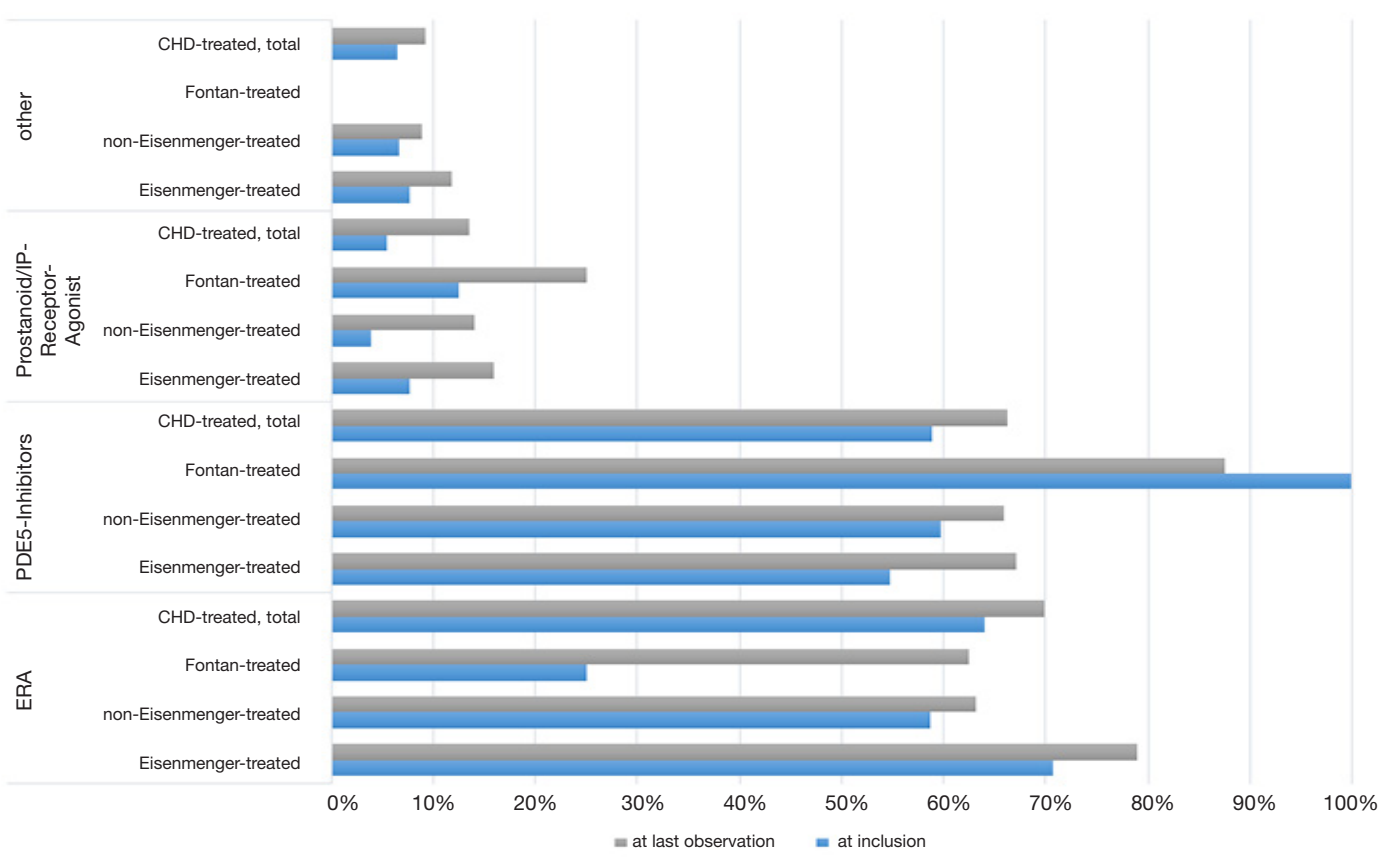

Figure 2 Targeted PAH medication for 674 PAH-CHD-patients in the COMPERA-CHD registry with at least one follow-up, until last follow-up in 2020, shown as percentage depending on diagnosis and targeted PAH medication. CHD, congenital heart disease; ERA, Endothelin receptor antagonist; Non-EM-PAH, patients with congenital heart disease-associated pulmonary arterial hypertension but without Eisenmenger syndrome; IP, selective prostacyclin receptor (IP receptor) agonist.

has increased by $13.4 \%$ (Table 1). Within this group the number of Eisenmenger patients rose by 39 (16.3\%). Of particular note is the fact that in the PAH-CHD group, the proportion of patients with "Non-Eisenmenger PAH" has risen substantially $(\mathrm{n}=45 ; 26.9 \%)$. In parallel, the number of participating centers increased by 13 (26.5\%). This may reflect a needed correction to a lack of awareness of the importance of systematic follow-up in patients with CHD, which has also been documented in contemporary studies $(2,14)$. In this respect, particular attention must be paid to the "Non-Eisenmenger PAH" group in the years and decades to come.

\section{Congenital heart anomalies and demographic data}

Unlike other registries, COMPERA-CHD includes a high proportion of patients with complex CHD ( $\mathrm{n}=121 ; 17.8 \%)$. These patients are often excluded from clinical trials. In addition, pre-tricuspid and post-tricuspid septal defects were enrolled; these typically account for the majority of CHD. Another unique feature of COMPERA-CHD is that patients with Eisenmenger's syndrome who were not on targeted PAH therapy, as well as patients with Fontan circulation who were on targeted PAH therapy for PVD, were also included.

The age of patients included in COMPERA-CHD was $45 \pm 17$ years. Notably, this is 5 to 13 years older than the patient ages in other international registries on $\mathrm{PAH}$ CHD but younger than the patient ages in large registries on other forms of PAH (15-20). The sex ratio, with a preponderance of females, is similar to that of all other registries, with the exception of the German National Registry for CHD, in which more males than females with PAH-CHD were reported (Table 1) (21).

At the time of inclusion in the COMPERA-CHD registry, the majority of PAH-CHD patients were in functional class III, correlating with the limited $6 \mathrm{MWD}$ of $369 \pm 121 \mathrm{~m}$, which is similar to limitations reported in other registries. There were no relevant differences in the data from 2020 compared with the previous registry data reported in 2018 for the following parameters: CHD diagnosis, age, sex, functional class, or 6MWD (Tables 1,2).

However, particularly striking is the high proportion of patients with cardiac and non-cardiac comorbidities, an entity that has been largely disregarded in previous studies and analyses. The analysis of the 2020 data revealed for the 


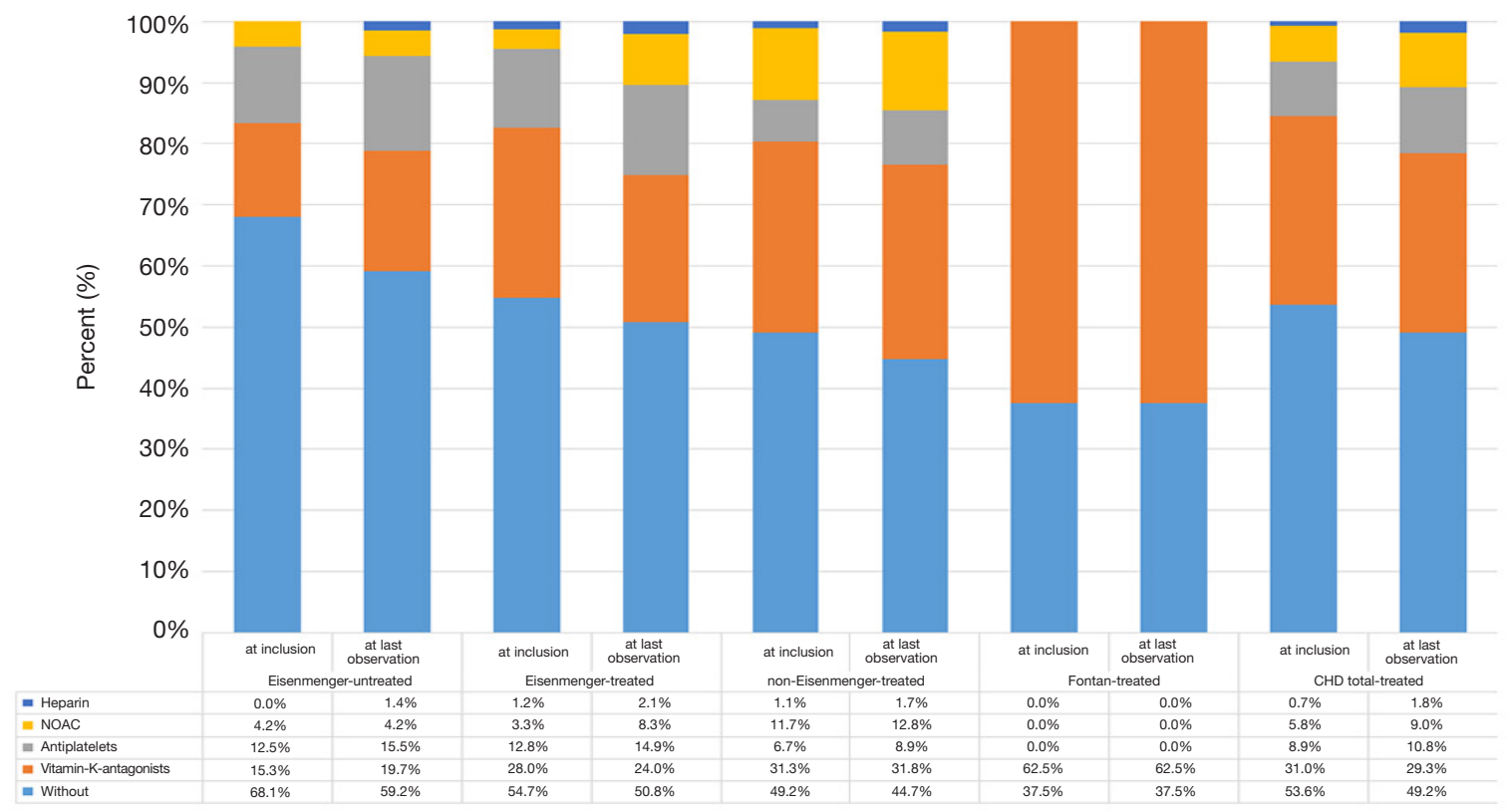

Figure 3 Percentage of anticoagulant use in 674 PAH-CHD patients in the COMPERA-CHD registry with at least one follow-up until last follow-up in 2020 at the time of inclusion and at last available follow-up in the COMPERA-CHD registry. NOAC, Non-Vitamin K antagonist oral anticoagulants; non-Eisenmenger-PAH, patients with congenital heart disease-associated pulmonary arterial hypertension but without Eisenmenger syndrome.

first time in the COMPERA-CHD-registry comorbidities of clinical relevance in 289 of 656 patients $(44.1 \%)$, in particular coronary artery disease, arterial hypertension, thromboembolism, sleep apnea syndrome, diabetes mellitus, and thyreopathies. Some of these comorbidities certainly correspond to the usual distribution in the normal population. For thyreopathies, a correlation with the presence of trisomy 21 ( $n=100$ of 448$)$, the use of amiodarone ( $\mathrm{n}=28$ of 568), and the fact that Germany is an iodine-deficient area are potentially relevant factors (22).

\section{Targeted medical PAH treatment}

In COMPERA-CHD, all available targeted PAH drugs have been used, including ERAs, PDE5i, prostanoids, prostacyclin receptor agonists, and sGCs (Figure 2).

At the time of the last follow-up (data base freeze 09/2020), in COMPERA-CHD ERAs ( $\mathrm{n}=454)$ and PDE5i $(n=416)$ were preferentially administered in all groups. Prostanoids or an sGC stimulator were used in only a minority of patients; 14 (2.3\%). Some preparations (e.g., the ERA sitaxentan) were withdrawn during the observation period and replaced by others. Calcium channel blockers are currently used only exceptionally $(n=26)$ in responders after hemodynamic vasoreactivity testing.

At the time of inclusion in COMPERA-CHD, the majority of all CHD patients received monotherapy $(69 \%)$, and distinctly less frequently combination therapy (31\%). This is partly attributable to the fact that many patients were enrolled in the registry more than 10 years ago. At least at that time, there was a tendency to initiate combination therapy only in cases of symptomatic deterioration or when predefined and center-specific treatment goals were not achieved. This is in contrast to the current tendency to favor early combination therapy of two or even more PAH-active drugs, at least for some forms of PAH.

The reluctance to using combination therapies in PAHCHD may originate from the fact that, particularly in Eisenmenger syndrome, evidence-based and consensusdriven treatment algorithms are not available, and there is no robust evidence to support the use of PAH combination therapy (23). However, in recent years, some study data and reports have suggested that adults with CHD may also benefit from targeted combination therapy (7,24-28). Accordingly, a more aggressive approach is also reflected in the COMPERA-CHD data, as the percentage of initially monotherapy (41\%) versus combination therapy 
Table 3 Assignment of baseline data to different PAH-CHD subgroups depending on diagnosis and treatment status at final assessment. A total of 190 patients could not be clearly assigned to any of the subgroups

\begin{tabular}{|c|c|c|c|c|c|c|c|c|c|c|}
\hline \multirow[t]{2}{*}{ Baseline data } & \multicolumn{2}{|c|}{ PAH-CHD total } & \multicolumn{2}{|c|}{$\begin{array}{l}\text { EM without targeted } \\
\text { PAH medication }\end{array}$} & \multicolumn{2}{|c|}{$\begin{array}{c}\text { EM with targeted } \mathrm{PAH} \\
\text { medication }\end{array}$} & \multicolumn{2}{|c|}{$\begin{array}{c}\text { Non-EM-PAH with } \\
\text { targeted PAH medication }\end{array}$} & \multicolumn{2}{|c|}{$\begin{array}{c}\text { Fontan with targeted } \\
\text { PAH medication }\end{array}$} \\
\hline & $\mathrm{n}$ & $\%$ & $\mathrm{n}$ & $\%$ & $\mathrm{n}$ & $\%$ & $\mathrm{n}$ & $\%$ & $\mathrm{n}$ & $\%$ \\
\hline Total & \multicolumn{2}{|c|}{769} & \multicolumn{2}{|c|}{79} & \multicolumn{2}{|c|}{279} & \multicolumn{2}{|c|}{212} & \multicolumn{2}{|c|}{9} \\
\hline Age & \multicolumn{2}{|c|}{$45.3 \pm 16.8$} & \multicolumn{2}{|c|}{$40.2 \pm 15.2$} & \multicolumn{2}{|c|}{$43.2 \pm 15.0$} & \multicolumn{2}{|c|}{$48.4 \pm 17.7$} & \multicolumn{2}{|c|}{$36.0 \pm 11.6$} \\
\hline Female & 512 & 66.6 & 54 & 68.4 & 187 & 67.0 & 142 & 67.0 & 4 & 44.4 \\
\hline \multicolumn{11}{|l|}{ FC } \\
\hline Unknown & 92 & 12.0 & 30 & 38.9 & 24 & 8.6 & 20 & 9.4 & 4 & 44.4 \\
\hline IV & 29 & 3.8 & 3 & 3.8 & 12 & 4.3 & 4 & 1.9 & 0 & 0 \\
\hline $\begin{array}{l}\text { 6-minute } \\
\text { walk distance } \\
\text { (meters) }\end{array}$ & \multicolumn{2}{|c|}{$369 \pm 121(n=508)$} & \multicolumn{2}{|c|}{$386 \pm 120(n=28)$} & \multicolumn{2}{|c|}{$354 \pm 123(n=192)$} & \multicolumn{2}{|c|}{$390 \pm 120(n=145)$} & \multicolumn{2}{|c|}{$464 \pm 40(n=3)$} \\
\hline
\end{tabular}

EM, Eisenmenger syndrome; FC, Functional class according to Perloff; Non-EM-PAH, patients with congenital heart disease-associated pulmonary arterial hypertension but without Eisenmenger syndrome; $\mathrm{PAH}$, pulmonary arterial hypertension; PAH-CHD, Congenital heart disease-associated pulmonary arterial hypertension; PAH medication, targeted medication to treat PAH.

(53\%) shifted during the observation period in favor of combination therapy.

\section{Additive and supportive medical treatment}

It is noteworthy that a large proportion of the included patients received supplementary drug treatment in addition to targeted PAH medication; preferably diuretics, betablockers, ACE inhibitors or AT blockers, digitalis, or class III antiarrhythmics. The application of these drugs should not be underestimated, as their use can be critical, especially in cyanotic patients, including Eisenmenger's syndrome, and side effects can be expected. In particular, lowering of peripheral vascular resistance with consecutive deepening of cyanosis may occur with ACE inhibitors or AT blockers; hemoconcentration and enhancement of hyperuricemia may occur with thiazide diuretics; and higher-degree heart block may occur with beta blockers or digitalis $(29,30)$.

As anticoagulants, vitamin $\mathrm{K}$ antagonists or direct-acting, non-vitamin $\mathrm{K}$ antagonists (NOAC) and platelet inhibitors were used (Figure 3). The indication for anticoagulants in
$\mathrm{PAH}$ is still under debate, in particular in Eisenmenger syndrome, where there is, on the one hand, an increased risk of bleeding, and, on the other hand, an increased risk of thrombosis, from abnormalities in platelet function, platelet count, and other coagulation parameters (29,31-35). Because data are insufficient to determine whether oral anticoagulation has a positive effect on morbidity and mortality in Eisenmenger syndrome, current ACHD guidelines do not recommend the routine use of oral anticoagulants and explicitly address potential adverse effects $(7,36,37)$.

The COMPERA-CHD data also reflect the fact that in Eisenmenger syndrome, oral anticoagulants are currently restricted to specific indications in patients without significant hemoptysis: the vast majority of PAH-CHD patients $(53.6 \%)$ did not receive anticoagulants. However, while at the time of initial data entry in COMPERA-CHD, patients with Eisenmenger syndrome were mostly not anticoagulated, the number of anticoagulated patients has increased since. This may be explained by complications in the long-term course (e.g., atrial arrhythmias, 
thromboembolism, mechanical valve replacement) resulting in the need to use these drugs. Surprisingly, in both the Eisenmenger groups, with and without targeted PAH therapy, up to $15 \%$ of patients were treated with antiplatelets, despite the fact that almost all of them have a genuine disorder of platelet number and function (38).

In contrast, the small group of Fontan patients was predominantly treated with anticoagulants because of the high rate of thromboembolic events (up to $33 \%$ ) expected due to the particular hemodynamic and coexistent coagulopathy and hypercoagulation states commonly seen after post-Fontan operation (37,39-42).

\section{Strengths and limitations}

Strengths of the presented registry study include the relatively large sample size of prospectively registered patients with PAH-CHD, the "real-world" conditions, and the long observation period.

Limitations include the voluntary nature of a registry, although COMPERA-CHD did enroll patients prospectively and consecutively, and implemented various control measures to ensure high data quality. Statistical measures to reduce confounding factors, such as propensity score matching or multivariable risk-adjusted modeling, were not performed. Therefore, group differences at baseline must be considered when evaluating all results and conclusions.

Finally, it should be emphasized that the registry study was performed at tertiary care centers for ACHD or for $\mathrm{PAH}$. Thus, the distribution of patients in terms of type and severity of CHD does not resemble the typical patient population seen outside of these centers. Generalization of the conclusions and transfer of the findings to patients of different countries or cultures is potentially problematic. Further studies are needed in this regard.

\section{Conclusions}

Based on real-world data from the international COMPERA-CHD registry, our updated analysis provides a comprehensive overview about current management modalities and treatment concepts for PAH-CHD patients. While individual therapy was dependent on the underlying CHD, there was an overall trend towards more aggressive treatment strategies and combination therapies.

Hence, our data may help to uncover even closer associations between the type and severity of the particular
CHD, treatment status, and outcome, and may also serve as a basis for further research. In this context, detailed longterm survival analyses should clarify the mortality in each patient group related to the treatment regimen. Potentially, registry data may continue to advance the management of PAH-CHD patients and aid in the development of screening and treatment protocols, and thereby further improve the management of affected individuals.

\section{Acknowledgments}

The authors are indebted to the COMPERA investigators and their staff. We explicitly thank Dr. Claudia S. Copeland for the professional editing of the final draft of the manuscript.

Funding: COMPERA is funded by unrestricted grants from Acceleron, Actelion Pharmaceuticals (Janssen), Bayer, OMT and GSK. These companies were not involved in data analysis or the writing of this manuscript.

\section{Footnote}

Provenance and Peer Review: This article was commissioned by the Guest Editors (Yskert von Kodolitsch, Harald Kaemmerer, Koichiro Niwa) for the series "Current Management Aspects in Adult Congenital Heart Disease (ACHD): Part IV" published in Cardiovascular Diagnosis and Therapy. The article has undergone external peer review.

Conflicts of Interest: All authors have completed the ICMJE uniform disclosure form (available at https:// dx.doi.org/10.21037/cdt-21-351). The series "Current Management Aspects in Adult Congenital Heart Disease (ACHD): Part IV" was commissioned by the editorial office without any funding or sponsorship. Dr. DH reports non-financial support from Actelion, BoehringerIngelheim, and Shire, outside the submitted work; Dr. DP reports personal fees from Actelion, Biogen, Aspen, Bayer, Boehringer Ingelheim, Daiichi Sankyo, and Sanofi, outside the submitted work; Dr. MD reports personal fees from Actelion, Bayer, GSK and MSD, outside the submitted work; Dr. HAG reports personal fees from Actelion, Bayer, Gilead, GSK, MSD, Pfizer and United Therapeutics, outside the submitted work; Dr. MG reports personal fees from Actelion, Bayer and GSK, outside the submitted work; Dr. MMH reports personal fees from Acceleron, Actelion, Bayer, MSD and Pfizer, outside the submitted work; Dr. CDV reports personal fees from Actelion, Bayer, 
GSK, MSD, Pfizer, and United Therapeutics, outside the submitted work; Dr. RE reports personal fees from Actelion, Boehringer Ingelheim, OMT, Bayer, and Berlin Chemie; grants from Actelion and Boehringer Ingelheim, outside the submitted work; Dr. MH reports grants and personal fees from Actelion, personal fees from Bayer, Berlin Chemie, Boehringer Ingelheim, GSK, Janssen, Novartis and MSD, outside the submitted work; Dr. MH reports personal fees from Acceleron, Actelion, AstraZeneca, Bayer, BERLIN CHEMIE, GSK, MSD, Novartis and OMT, outside the submitted work; Dr. HW reports personal fees from Action, Bayer, Biotest, Boehringer, GSK, Pfizer, and Roche, outside the submitted work; Dr. DS reports personal fees from Actelion, Bayer, and GSK, outside the submitted work; Dr. LS reports personal fees from Actelion, Bayer, and MSD, outside the submitted work; Dr. SU reports grants from Swiss National Science Foundation, Zurich Lung, Swiss Lung, and Orpha Swiss, grants and personal fees from Actelion SA/Johnson \& Johnson, Switzerland, and MSD Switzerland, outside the submitted work; Dr. TJL reports personal fees from Actelion, Janssen-Cilag, BMS, MSD, and OMT GmbH, outside the submitted work; Dr. LB reports personal fees from Actelion, outside the submitted work; Dr. MC reports personal fees from Boehringer Ingelheim Pharma GmbH, Roche Pharma, and Boehringer Ingelheim, outside the submitted work; Dr. HW reports personal fees from Boehringer Ingelheim, and Roche, outside the submitted work. Dr. EG reports personal fees from Actelion, Janssen, Bayer, MSD, Bial, OrPha Swiss GmbH, OMT and Medscape, outside the submitted work; Dr. SR reports personal fees from Actelion, Bayer, GSK, Pfizer, Novartis, Gilead, MSD, and United Therapeutics, outside the submitted work. The authors have no other conflicts of interest to declare.

Ethical Statement: The authors are accountable for all aspects of the work in ensuring that questions related to the accuracy or integrity of any part of the work are appropriately investigated and resolved. The registry has been approved by the institutional review boards of all contributing centers and written informed consent was obtained from all participating patients before the start of documentation (lead ethics committee at Technical University Dresden, Germany: approval EK 129052007 as of 22 May 2007). The study was conducted in accordance with the Declaration of Helsinki (as revised in 2013).

Open Access Statement: This is an Open Access article distributed in accordance with the Creative Commons Attribution-NonCommercial-NoDerivs 4.0 International License (CC BY-NC-ND 4.0), which permits the noncommercial replication and distribution of the article with the strict proviso that no changes or edits are made and the original work is properly cited (including links to both the formal publication through the relevant DOI and the license). See: https://creativecommons.org/licenses/by-nc-nd/4.0/.

\section{References}

1. Neidenbach R, Niwa K, Oto O, et al. Improving medical care and prevention in adults with congenital heart diseasereflections on a global problem-part I: development of congenital cardiology, epidemiology, clinical aspects, heart failure, cardiac arrhythmia. Cardiovasc Diagn Ther 2018;8:705-15.

2. Neidenbach R, Achenbach S, Andonian C, et al. Systematic assessment of health care perception in adults with congenital heart disease in Germany. Cardiovasc Diagn Ther 2021;11:481-91.

3. Mandalenakis Z, Giang KW, Eriksson P, et al. Survival in Children With Congenital Heart Disease: Have We Reached a Peak at 97\%? J Am Heart Assoc 2020;9:e017704.

4. Perloff JK, Warnes CA. Challenges posed by adults with repaired congenital heart disease. Circulation 2001;103:2637-43.

5. Kaemmerer H, Mebus S, Schulze-Neick I, et al. The adult patient with eisenmenger syndrome: a medical update after dana point part I: epidemiology, clinical aspects and diagnostic options. Curr Cardiol Rev 2010;6:343-55.

6. Hoeper MM, Apitz C, Grünig E, et al. Targeted therapy of pulmonary arterial hypertension: Updated recommendations from the Cologne Consensus Conference 2018. Int J Cardiol 2018;272S:37-45.

7. Kaemmerer H, Apitz C, Brockmeier K, et al. Pulmonary hypertension in adults with congenital heart disease: Updated recommendations from the Cologne Consensus Conference 2018. Int J Cardiol 2018;272S:79-88.

8. Kaemmerer H, Gorenflo M, Huscher D, et al. Pulmonary Hypertension in Adults with Congenital Heart Disease: Real-World Data from the International COMPERACHD Registry. J Clin Med 2020;9:1456.

9. Coats AJ, Shewan LG. Statement on authorship and publishing ethics in the International Journal of Cardiology. Int J Cardiol 2011;153:239-40.

10. van Riel AC, Schuuring MJ, van Hessen ID, et al. 
Contemporary prevalence of pulmonary arterial hypertension in adult congenital heart disease following the updated clinical classification. Int J Cardiol 2014;174:299-305.

11. van Riel AC, Blok IM, Zwinderman AH, et al. Lifetime Risk of Pulmonary Hypertension for All Patients After Shunt Closure. J Am Coll Cardiol 2015;66:1084-6.

12. van der Velde ET, Vriend JW, Mannens MM, et al. CONCOR, an initiative towards a national registry and DNA-bank of patients with congenital heart disease in the Netherlands: rationale, design, and first results. Eur J Epidemiol 2005;20:549-57.

13. Kempny A, Dimopoulos K, Gatzoulis MA. Declining incidence and prevalence of Eisenmenger syndrome in the developed world: a triumph of modern medicine. Heart 2017;103:1313-4.

14. Seidel L, Nebel K, Achenbach S, et al. Facts about the General Medical Care of Adults with Congenital Heart Defects: Experience of a Tertiary Care Center. J Clin Med 2020;9:1943.

15. Ghofrani HA, Distler O, Gerhardt F, et al. Treatment of pulmonary arterial hypertension (PAH): updated Recommendations of the Cologne Consensus Conference 2011. Int J Cardiol 2011;154 Suppl 1:S20-33.

16. Favilli S, Spaziani G, Ballo P, et al. Advanced therapies in patients with congenital heart disease-related pulmonary arterial hypertension: results from a long-term, single center, real-world follow-up. Intern Emerg Med 2015;10:445-50.

17. Alonso-Gonzalez R, Lopez-Guarch CJ, SubiranaDomenech MT, et al. Pulmonary hypertension and congenital heart disease: An insight from the REHAP National Registry. Int J Cardiol 2015;184:717-23.

18. Jansa P, Jarkovsky J, Al-Hiti H, et al. Epidemiology and long-term survival of pulmonary arterial hypertension in the Czech Republic: a retrospective analysis of a nationwide registry. BMC Pulm Med 2014;14:45.

19. Van de Bruaene A, Delcroix M, Pasquet A, et al. The Belgian Eisenmenger syndrome registry: implications for treatment strategies? Acta Cardiol 2009;64:447-53.

20. Idrees M, Al-Najashi K, Khan A, et al. Pulmonary arterial hypertension in Saudi Arabia: Patients' clinical and physiological characteristics and hemodynamic parameters. A single center experience. Ann Thorac Med 2014;9:209-15.

21. Diller GP, Körten MA, Bauer UM, et al. Current therapy and outcome of Eisenmenger syndrome: data of the German National Register for congenital heart defects. Eur Heart J 2016;37:1449-55.
22. Rosenkranz S, Howard LS, Gomberg-Maitland M, et al. Systemic Consequences of Pulmonary Hypertension and Right-Sided Heart Failure. Circulation 2020;141:678-93.

23. Nashat H, Kempny A, Harries C, et al. A single-centre, placebo-controlled, double-blind randomised cross-over study of nebulised iloprost in patients with Eisenmenger syndrome: A pilot study. Int J Cardiol 2020;299:131-5.

24. D'Alto M, Constantine A, Balint OH, et al. The effects of parenteral prostacyclin therapy as add-on treatment to oral compounds in Eisenmenger syndrome. Eur Respir J 2019;54:1901401.

25. Durongpisitkul K, Chungsomprasong P, Vijarnsorn C, et al. Improved low-risk criteria scores for combination therapy of sildenafil and generic bosentan in patients with congenital heart disease with severe pulmonary hypertension: A prospective open label study. JRSM Cardiovasc Dis 2021;10:2048004020982213.

26. D'Alto M, Romeo E, Argiento P, et al. Bosentan-sildenafil association in patients with congenital heart diseaserelated pulmonary arterial hypertension and Eisenmenger physiology. Int J Cardiol 2012;155:378-82.

27. Iversen K, Jensen AS, Jensen TV, et al. Combination therapy with bosentan and sildenafil in Eisenmenger syndrome: a randomized, placebo-controlled, doubleblinded trial. Eur Heart J 2010;31:1124-31.

28. Manes A, Palazzini M, Leci E, et al. Current era survival of patients with pulmonary arterial hypertension associated with congenital heart disease: a comparison between clinical subgroups. Eur Heart J 2014;35:716-24.

29. Grünig E, Benjamin N, Krüger U, et al. General measures and supportive therapy for pulmonary arterial hypertension: Updated recommendations from the Cologne Consensus Conference 2018. Int J Cardiol 2018;272S:30-6.

30. Oechslin E, Mebus S, Schulze-Neick I, et al. The Adult Patient with Eisenmenger Syndrome: A Medical Update after Dana Point Part III: Specific Management and Surgical Aspects. Curr Cardiol Rev 2010;6:363-72.

31. Galiè N, Hoeper MM, Humbert M, et al. Guidelines for the diagnosis and treatment of pulmonary hypertension: the Task Force for the Diagnosis and Treatment of Pulmonary Hypertension of the European Society of Cardiology (ESC) and the European Respiratory Society (ERS), endorsed by the International Society of Heart and Lung Transplantation (ISHLT). Eur Heart J 2009;30:2493-537.

32. Olsson KM, Delcroix M, Ghofrani HA, et al. Anticoagulation and survival in pulmonary arterial 
hypertension: results from the Comparative, Prospective Registry of Newly Initiated Therapies for Pulmonary Hypertension (COMPERA). Circulation 2014;129:57-65.

33. Kempny A, Hjortshøj CS, Gu H, et al. Predictors of Death in Contemporary Adult Patients With Eisenmenger Syndrome: A Multicenter Study. Circulation 2017;135:1432-40.

34. Hjortshøj CMS, Kempny A, Jensen AS, et al. Past and current cause-specific mortality in Eisenmenger syndrome. Eur Heart J 2017;38:2060-7.

35. Lill MC, Perloff JK, Child JS. Pathogenesis of thrombocytopenia in cyanotic congenital heart disease. Am J Cardiol 2006;98:254-8.

36. Baumgartner H, De Backer J. The ESC Clinical Practice Guidelines for the Management of Adult Congenital Heart Disease 2020. Eur Heart J 2020;41:4153-4.

37. Stout KK, Daniels CJ, Aboulhosn JA, et al. 2018 AHA/ ACC Guideline for the Management of Adults With

Cite this article as: Kaemmerer AS, Gorenflo M, Huscher D, Pittrow D, Ewert P, Pausch C, Delcroix M, Ghofrani HA, Hoeper MM, Kozlik-Feldmann R, Skride A, Stähler G, Vizza CD, Jureviciene E, Jancauskaite D, Gumbiene L, Ewert R, Dähnert I, Held M, Halank M, Skowasch D, Klose H, Wilkens H, Milger K, Jux C, Koestenberger M, Scelsi L, Brunnemer E, Hofbeck M, Ulrich S, Vonk Noordegraaf A, Lange TJ, Bruch L, Konstantinides S, Claussen M, Löffler-Ragg J, Wirtz H, Apitz C, Neidenbach R, Freilinger S, Nemes A, Opitz C, Grünig E, Rosenkranz S. Medical treatment of pulmonary hypertension in adults with congenital heart disease: updated and extended results from the International COMPERA-CHD Registry. Cardiovasc Diagn Ther 2021;11(6):1255-1268. doi: 10.21037/cdt-21-351
Congenital Heart Disease: Executive Summary: A Report of the American College of Cardiology/American Heart Association Task Force on Clinical Practice Guidelines. J Am Coll Cardiol 2019;73:1494-563.

38. Pujol C, Stöckl A, Mebus S, et al. Value of Rotational Thromboelastometry and Impedance Aggregometry for Evaluating Coagulation Disorders in Patients With Cyanotic and Nongenetic Congenital Heart Disease. Am J Cardiol 2019;123:1696-702.

39. Balling $G$, Vogt $M$, Kaemmerer $H$, et al. Intracardiac thrombus formation after the Fontan operation. J Thorac Cardiovasc Surg 2000;119:745-52.

40. Balling G. Fontan Anticoagulation: A Never-Ending Debate? J Am Coll Cardiol 2016;68:1320-2.

41. Khairy P, Poirier N, Mercier LA. Univentricular heart. Circulation 2007;115:800-12.

42. Viswanathan S. Thromboembolism and anticoagulation after Fontan surgery. Ann Pediatr Cardiol 2016;9:236-40. 